\title{
Peri-Procedural Protocols for Interventional Pain Management TeChniques: A Survey of US Pain Centers
}

\author{
Shihab U. Ahmed, MD, MPH, William Tonidandel DMD, MD, Jason Trella, Nicole M. Martin BS, and \\ Yuchiao Chang, PhD
}

\begin{abstract}
Background : Interventional techniques are now an integral part of chronic pain management. As new procedures are arising at a rapid pace, decisions regarding patient safety and comfort are becoming more challenging. No peri-procedural consensus protocol currently addresses issues such as 1 . nulla per os (NPO) status, 2. sedation, 3. monitoring, or 4 . recovery. In establishing safety guidelines for interventional pain procedures, the knowledge of current peri-procedural protocols is required.

Objective: To survey interventional pain practices and to obtain current peri-procedural protocols.
\end{abstract}

Design: We faxed a one-page ques- tionnaire to 105 United States pain practices identified using the directory of the American Pain Society. Fifty-seven academic and private pain practices (54\%) responded and were included in the analysis.

Results: Monitoring devices such as electrocardiogram (EKG), blood pressure, and pulse oximetry are not universally employed for cervical or lumbar spinal procedures. Even procedures that are often performed by anesthesiologists in operating rooms, such as Bier blocks, are not monitored in a uniform manner when performed in pain clinics. Establishment of intravenous access for procedures also varies among practitioners. Most (72\%) practices had treated pa- tients with vasovagal reactions over the past 12 months, but only $42 \%$ had simulated cardiac arrests to prepare for these situations.

Conclusion: While various trends in peri-procedural care are observable, standards of care are not well established. In order to minimize complications associated with interventional pain management techniques, the pain management community should agree on safety guidelines for all procedures, much as these advocated by the American Society of Anesthesiology for surgical anesthetic care.

Keywords: Interventional pain management, nerve block, sedation, monitoring, recovery
Interventional pain management techniques (IPMTs) are becoming increasingly important in the comprehensive management of chronic pain. Although morbidity from these procedures is generally low $(1,2)$ sequelae such as permanent neurological damage and death have been reported (3-5). IPMTs are currently performed in various settings, including physicians' offices, ambulatory surgery centers, and operating rooms. Pain practitioners from both anesthesia and nonanesthesia backgrounds must now provide peri-procedural care for patients that have IPMTs. Decisions must be made regarding NPO status, peri-operative mon-

From: Massachusetts General Hospital Pain Center, Boston, MA, St. Peter's Hospital, Helena, MT; and Harvard Medical School, Boston, MA

Address Correspondence:

Shihab U. Ahmed, MD, Massachusetts General Hospital Pain Center, WACC 324, 15 Parkman Street, Boston, MA 02114.

Disclaimer: There was no external funding in preparation of this manuscript.

Conflict of Interest: None

Acknowledgement:

Manuscript received on 1/24/2005

Revision submitted on 3/3/2005

Accepted for publication on 3/9/2005 itoring, and post-procedural care. Variations among settings and providers have resulted in heterogeneous monitoring, sedation, and recovery protocols.

The field of surgical anesthesia has well-established guidelines for pre-, intra-, and postoperative care (6-8). For a surgical procedure, the anesthesiologist usually performs the role of perioperative physician, assuming responsibility for patient comfort and safety. Although many anesthesiologists in pain management follow the guidelines suggested by the American Society of Anesthesiology (ASA) with regard to NPO status and routine monitoring, to our knowledge the field of pain medicine has no established standards. The International Spinal Intervention Society (ISIS) has published a series of guidelines for selected interventions (9), but no comprehensive protocol addresses patient safety issues for the full breadth of pain procedures. One issue of particular interest is that of peri-procedural sedation. For patient comfort, some physicians dispense oral (PO) sedatives before, and intravenous (IV) sedatives during procedures. This practice raises questions regarding airway protection and potential for neural injury during procedures.

The purpose of this study was to examine current standards at both academic and private pain centers regarding preprocedural (nulla per os status, pre-medication, intravenous line placement), intra-procedural (blood pressure, electrocardiogram, pulse oximetry, IV sedation), and post-procedural (recovery time and monitoring) care, as well as preparedness for complications (particularly cardiopulmonary arrest). By highlighting discrepancies among practices, we hope to initiate a discussion in the pain community so that guidelines for peri-procedural care may be established.

\section{Methods}

Questions were developed by one of the authors of this report, Shihab Ahmed, $\mathrm{MD}$; three experienced physicians reviewed a draft and offered suggestions. The survey consisted of 134 questions of 16 commonly performed pain procedures. The questions were divided into three sections (Appendix 1). The first section sought to characterize partici- 
pating pain practices. The second section examined current protocols for preprocedural NPO status, pre-procedural oral sedation, intra-procedural monitoring and intravenous sedation, and postprocedural recovery. The final section focused on pre-, intra-, and post-procedural complications that had occurred in the 12 months prior to survey completion.

Pain practices were randomly selected from the 2002 member directory of the American Pain Society (APA); clinics from all 50 states were included. We faxed surveys with cover letters to a total of 105 clinics. No monetary or other compensation was offered for completing and returning the surveys. Each practice not responding within two weeks received a second copy by fax.

Descriptive statistics have been used to summarize the survey results. Not all respondents answered every question in the survey. Thus, all statistics (mean values for continuous variables and percentage response rates for categorical variables) have been calculated for the subse of practices that responded to each individual question.

\section{RESULTS}

\section{Demographics}

Completed questionnaires were returned by 61 practices, and four were excluded from the analysis due to unanswered questions or ambiguous responses. Thus, the response rate was 54\% (57/ 105). Most of the responding pain practices (RPPs) were anesthesiology-based ( $\mathrm{n}=$ $45,79 \%$ ); other specialties included physical medicine and rehabilitation (PMR) (n $=3,5.3 \%$ ), combined anesthesiology and PMR ( $\mathrm{n}=2,3.5 \%)$, anesthesiology and neurology $(\mathrm{n}=1,1.8 \%)$, and anesthesiology with an unspecified specialty $(\mathrm{n}=1$, $1.8 \%$ ). Five practices did not indicate their specialties. Most RPPs $(n=36,63 \%)$ were in academia; others were private practices $(\mathrm{n}=11,19.3 \%)$ or combined private/ academic practices $(\mathrm{n}=10,17.5 \%)$.

\section{Peri-Procedural Practices Pain Clinics}

\section{NPO orders and sedation}

For most procedures, the majority of the RPPs have adopted NPO policies (mean $=74 \%$ for all procedures). NPO is especially common for spinal cord stimulation trials (95\%) and cervical and lumbar discographies (94\% for each); it is less common for intercostal nerve blocks (58\%) and peripheral nerve blocks (46\%). The median time for NPO status is six hours prior to the scheduled procedures (ranges: 2-12 hours for most procedures, 4-12 hours for IV lidocaine administration).

Few RPPs routinely dispense medication to be taken by mouth (PO) prior to procedures $($ mean $=7 \%$ for all procedures). Procedures for which PO sedatives are most commonly given are peripheral nerve blocks (12\%), Bier blocks (11\%), and intercostal nerve blocks (10\%). Seven RPPs indicated that they use diazepam (Valium) as their PO medication of choice.

Intravenous (IV) sedation practices for IPMTs vary widely ( mean $=64 \%$ for all procedures). IV sedation is typically given for cervical and lumbar discography ( $90 \%$ and $92 \%$, respectively) and spinal cord stimulator trials (90\%). Less than half of RPPs use IV sedation for lumbar epidural steroid injections $(46 \%)$, stellate ganglion blocks (46\%), intercostal nerve blocks (44\%), and peripheral nerve blocks $(30 \%)$. These results are summarized in Table 1.

\section{Patient monitoring and IV access}

The majority of RPPs $(>80 \%)$ use non-invasive blood pressure monitors (NIBP) during procedures, and pulse oximetry is also common $(>70 \%)$. For peripheral nerve blocks, these devices are less frequently utilized; slightly over half the RPPs surveyed employ NIBP (56\%) and pulse oximetry (52\%) during such procedures. Electrocardiogram (EKG) monitoring is most commonly used during IV lidocaine administrations (89\%). Placement of IV lines varies by procedure. Over $90 \%$ of practitioners place lines for lumbar and cervical discographies (94\% for each), spinal cord stimulator trials (93\%), and continuous intrathecal drug delivery trials $(92 \%)$. Less than half of respondents place IV lines for intercostal nerve blocks (48\%), lumbar epidural steroid injections $(47 \%)$, and peripheral nerve blocks (33\%). These results are summarized in Table 1.

\section{Post-procedural recovery}

After completed procedures, patients generally spend time in the recovery area for observation. Over $85 \%$ of respondents send patients to the recovery area after most procedures; fewer do so after intercostal nerve blocks (78\%) and peripher- al nerve blocks (72\%). For post-procedural observation, 47 practices utilize nurses, two use physicians, and one employs medical assistants. Median recovery time is 30 minutes for all procedures (ranges: 10-90 minutes for Bier blocks and lumbar sympathetic blocks; 10-120 minutes for continuous intrathecal drug delivery trials and cervical and lumbar discographies; 10-180 minutes for spinal cord stimulator trials; and 10-60 minutes for all other procedures). RPPs cited no hospital admissions among their patients, with the exception of those admitted on a predetermined basis for continuous intrathecal drug delivery trials (18\% of respondents).

\section{Complications}

Vasovagal responses occur frequently among patients undergoing pain procedures. In the 12 months prior to survey completion, most RPPs (72\%) treated patients who experienced such reactions, with a mean 7.3 reactions occurring per practice $($ median $=5$; range $=1-50)$. Cardiopulmonary arrest ("coding") is rare. Only $6 \%$ of respondents have dealt with this situation in the previous year, with a mean of 4.3 affected patients per practice $($ median $=2$, range $=1-10)$. Less than half of RPPs (42\%) routinely perform "mock codes" or train their staff members in emergency procedures.

\section{Discussion}

Our survey illustrates that universal practice parameters have yet to be established for peri-procedural sedation, monitoring, and recovery for interventional pain management techniques. Surprisingly, some pain practices choose not to use basic monitors (BP, EKG, pulse oximetry) for procedures, such as Bier blocks, that are typically performed by anesthesiologists in the operating room. Furthermore, an ISIS guideline for cervical procedures such as facet joint injection states that no physiologic monitoring or intravenous access is required (9). By contrast, even minor surgical procedures are covered by ASA's basic monitoring standards (BP, EKG, pulse oximetry).

The majority of RPPs indicated that they adhere to the basic ASA monitoring standards during most procedures. NIBP and oxygen saturation monitoring is more common than EKG monitoring during these procedures. The selection of the monitors is somewhat arbitrary among our RPPs. Most practices (72\%) 
Table 1. Safety and comfort measures reported by responding pain practices

\begin{tabular}{|l|c|c|c|c|c|c|}
\hline \multicolumn{1}{|c|}{ Procedure } & $\begin{array}{c}\text { NPO } \\
\text { Status }\end{array}$ & NIBP & $\begin{array}{c}\text { Pulse } \\
\text { Oximetry }\end{array}$ & EKG & IV line & IV Sedation \\
\hline Cervical discogram & $94 \%$ & $96 \%$ & $94 \%$ & $81 \%$ & $94 \%$ & $90 \%$ \\
\hline Lumbar discogram & $94 \%$ & $97 \%$ & $94 \%$ & $82 \%$ & $94 \%$ & $92 \%$ \\
\hline Intrathecal catheter drug delivery trial & $83 \%$ & $96 \%$ & $89 \%$ & $82 \%$ & $92 \%$ & $71 \%$ \\
\hline Spinal cord stimulator trial & $95 \%$ & $95 \%$ & $91 \%$ & $85 \%$ & $93 \%$ & $90 \%$ \\
\hline Bier block & $79 \%$ & $95 \%$ & $87 \%$ & $84 \%$ & $87 \%$ & $62 \%$ \\
\hline IV lidocaine & $79 \%$ & $93 \%$ & $89 \%$ & $89 \%$ & $89 \%$ & $50 \%$ \\
\hline Cervical facet & $69 \%$ & $92 \%$ & $87 \%$ & $57 \%$ & $70 \%$ & $64 \%$ \\
\hline Lumbar facet & $68 \%$ & $89 \%$ & $83 \%$ & $56 \%$ & $64 \%$ & $66 \%$ \\
\hline Cervical ESI & $68 \%$ & $89 \%$ & $79 \%$ & $52 \%$ & $72 \%$ & $53 \%$ \\
\hline Lumbar ESI & $61 \%$ & $84 \%$ & $74 \%$ & $43 \%$ & $47 \%$ & $46 \%$ \\
\hline Radiofrequency cervical & $80 \%$ & $93 \%$ & $86 \%$ & $63 \%$ & $82 \%$ & $81 \%$ \\
\hline Radiofrequency lumbar & $79 \%$ & $92 \%$ & $85 \%$ & $65 \%$ & $81 \%$ & $79 \%$ \\
\hline Lumbar sympathetic block & $76 \%$ & $91 \%$ & $87 \%$ & $63 \%$ & $74 \%$ & $62 \%$ \\
\hline Stellate ganglion block & $77 \%$ & $91 \%$ & $83 \%$ & $56 \%$ & $75 \%$ & $46 \%$ \\
\hline Intercostal nerve block & $58 \%$ & $82 \%$ & $76 \%$ & $49 \%$ & $48 \%$ & $44 \%$ \\
\hline Peripheral nerve block & $46 \%$ & $56 \%$ & $52 \%$ & $32 \%$ & $33 \%$ & $30 \%$ \\
\hline
\end{tabular}

$\mathrm{NPO}=$ nothing by mouth, NIBP=noninvasive blood pressure monitoring, EKG=electrocardiogram, IV line=placement of intravenous line, $\mathrm{ESI}=$ epidural steroid injection, Radiofreq=denervation of the facet joints by radiofrequency lessoning of the cervical or lumbar spine.

have treated at least one patient with a vasovagal episode and $6 \%$ had to deal with a "code" situation in the 12 months prior to survey completion. It is surprising that only $42 \%$ of the RPPs routinely train their staff members in emergency management (i.e., codes).

Most pain practices issue NPO orders for interventional spine procedures, and nearly half do so for peripheral nerve blocks. The survey findings indicate that there is a correlation between the NPO status and the use of IV sedation for IPMTs among pain physicians (Table 1). This may reflect safety concerns related to airway protection during the procedure when sedation is being used for patient comfort.

Although sedation increases patient comfort, it may also raise the risk of complications. A patient who is fully alert can report adverse phenomena during a procedure such as paresthesias, cardiovascular symptoms, and central nervous system (CNS) symptoms. Because of the potential for nerve injury, patient feedback is critical during procedures such as injections via the transforaminal route, radiofrequency lesioning (RFL), discography, and intradiscal electrothermal therapy (IDET). Sedation may also limit assessment during diagnostic procedures such as spinal cord stimulator (SCS) lead placement, and discography. In these situations, suboptimal patient feedback may adversely affect long-term outcomes.

This study has obvious demographic limitations, so our findings might not represent the full spectrum of American pain practices. The overall response rate (including the four rejected responses) was $58 \%$, and we had limited means of determining how respondents differed from non-respondents. We sent questionnaires to equal numbers of private and academic practices, the latter responded more frequently. Four returned surveys were excluded from the analysis because of ambiguous answers. Completing the questionnaire required time and effort, and we provided no compensation. Participating practices likely had different levels of experience with the IPMTs, and we were unable to adjust for these differences.

In the interests of establishing a set of guidelines for peri-procedural care, future discussion should focus on several questions:

1) Should the invasiveness and anatomical site of the procedure dictate the protocol?

2) Should we use patient risk assessment (similar to ASA class) to set the protocol?
3) Should NPO status apply only for procedures that require IV sedation?

4) Should oral vs. IV sedation patients be treated differently?

5) Who should perform sedation, and what minimum certifications are necessary?

6) Who should provide post-procedural care?

7) For post-procedural care, should guidelines exist for mandatory recovery times, or should they be set according to individual patient needs?

These questions have no definitive answers but a committee of peers could make reasonable recommendations. We hope that our findings will encourage a concerted effort to develop protocols so that reliable standards of care can be established.

\section{ConcLusion}

While various trends in peri-procedural care are observable, standards of care are not well established. In order to minimize complications associated with interventional pain management techniques, the pain management community should agree on safety guidelines for all procedures, much as those advocated by the American Society of Anesthesiology for surgical anesthetic care. 
Appendix 1. Survey sent to pain practices consisted of 134 questions concerning 16 commonly performed pain procedures.

\begin{tabular}{|c|c|c|c|c|c|c|c|c|}
\hline \multicolumn{4}{|c|}{$\begin{array}{l}\text { Which of the following best describes your practice: } \\
\text { Please indicate all that apply for the following: }\end{array}$} & \multicolumn{2}{|c|}{$\square$ Anesthesia $\square$ PM \& I } & \multicolumn{2}{|c|}{ R $\square$ Neurology $\square$ Other: } & $\square$ Private $\square$ Academic \\
\hline Procedure: & NIBP & PulseOx & EKG & IV Line & NPO/Hours & IV Sedation/Med Used & PO Meds Prior/Meds & Recovery/Minutes/By Whom \\
\hline Cervical (C) ESI & $\mathrm{Y} / \mathrm{N}$ & $\mathrm{Y} / \mathrm{N}$ & $\mathrm{Y} / \mathrm{N}$ & $\mathrm{Y} / \mathrm{N}$ & $\mathrm{Y} / \mathrm{N} \_$hours & _ Meds & _Meds & _mins./__ \\
\hline Lumbar (L)ESI & $\mathrm{Y} / \mathrm{N}$ & $\mathrm{Y} / \mathrm{N}$ & $\mathrm{Y} / \mathrm{N}$ & $\mathrm{Y} / \mathrm{N}$ & $\mathrm{Y} / \mathrm{N} \_$hours & $\mathrm{Y} / \mathrm{N} \_$Meds & $\mathrm{Y} / \mathrm{N} \_$Meds & $\mathrm{Y} / \mathrm{N} \_$mins./_ \\
\hline Cervical Facet & $\mathrm{Y} / \mathrm{N}$ & $\mathrm{Y} / \mathrm{N}$ & $\mathrm{Y} / \mathrm{N}$ & $\mathrm{Y} / \mathrm{N}$ & $\mathrm{Y} / \mathrm{N} \_$_hours & $\mathrm{Y} / \mathrm{N} \_$Meds & $\mathrm{Y} / \mathrm{N} \_$Meds & $\mathrm{Y} / \mathrm{N} \_$mins./_ \\
\hline Lumbar Facet & $\mathrm{Y} / \mathrm{N}$ & $\mathrm{Y} / \mathrm{N}$ & $\mathrm{Y} / \mathrm{N}$ & $\mathrm{Y} / \mathrm{N}$ & $\mathrm{Y} / \mathrm{N}$ _ hours & Meds & Meds & __mins./_ \\
\hline Radiofrequency (C) & $\mathrm{Y} / \mathrm{N}$ & $\mathrm{Y} / \mathrm{N}$ & $\mathrm{Y} / \mathrm{N}$ & $\mathrm{Y} / \mathrm{N}$ & $\mathrm{Y} / \mathrm{N} \_$hours & $\mathrm{Y} / \mathrm{N} \_$Meds & $\mathrm{Y} / \mathrm{N} \_$___ Meds & $\mathrm{Y} / \mathrm{N} \_$mins./_ \\
\hline Radiofrequency (L) & $\mathrm{Y} / \mathrm{N}$ & $\mathrm{Y} / \mathrm{N}$ & $\mathrm{Y} / \mathrm{N}$ & $\mathrm{Y} / \mathrm{N}$ & $\mathrm{Y} / \mathrm{N}$ _ hours & $\mathrm{Y} / \mathrm{N} \_$Meds & Meds & $\mathrm{Y} / \mathrm{N} \_$mins./_ \\
\hline Discogram (C) & $\mathrm{Y} / \mathrm{N}$ & $\mathrm{Y} / \mathrm{N}$ & $\mathrm{Y} / \mathrm{N}$ & $\mathrm{Y} / \mathrm{N}$ & $\mathrm{Y} / \mathrm{N} \_$hours & _Meds & _Meds & $\mathrm{Y} / \mathrm{N} \_$mins./_ \\
\hline Discogram (L) & $\mathrm{Y} / \mathrm{N}$ & $\mathrm{Y} / \mathrm{N}$ & $\mathrm{Y} / \mathrm{N}$ & $\mathrm{Y} / \mathrm{N}$ & $\mathrm{Y} / \mathrm{N} \ldots$ hours & Meds & Meds & _mins./_ \\
\hline Bier Block & $\mathrm{Y} / \mathrm{N}$ & $\mathrm{Y} / \mathrm{N}$ & $\mathrm{Y} / \mathrm{N}$ & $\mathrm{Y} / \mathrm{N}$ & $\mathrm{Y} / \mathrm{N} \_$hours & Meds & $\mathrm{Y} / \mathrm{N} \_$Meds & _mins./_ \\
\hline IV Lidocaine & $\mathrm{Y} / \mathrm{N}$ & $\mathrm{Y} / \mathrm{N}$ & $\mathrm{Y} / \mathrm{N}$ & $\mathrm{Y} / \mathrm{N}$ & $\mathrm{Y} / \mathrm{N} \_$hours & Meds & $\mathrm{Y} / \mathrm{N} \_$Meds & $\mathrm{Y} / \mathrm{N} \_$mins./_ \\
\hline Stellate Ganglion & $\mathrm{Y} / \mathrm{N}$ & $\mathrm{Y} / \mathrm{N}$ & $\mathrm{Y} / \mathrm{N}$ & $\mathrm{Y} / \mathrm{N}$ & $\mathrm{Y} / \mathrm{N} \_$hours & $\mathrm{Y} / \mathrm{N} \_$Meds & Meds & _mins./_ \\
\hline Lumbar Sympathetic & $\mathrm{Y} / \mathrm{N}$ & $\mathrm{Y} / \mathrm{N}$ & $\mathrm{Y} / \mathrm{N}$ & $\mathrm{Y} / \mathrm{N}$ & $\mathrm{Y} / \mathrm{N} \_$hours & Meds & Meds & $\mathrm{Y} / \mathrm{N} \_$mins./_ \\
\hline Peripheral Nerve & $\mathrm{Y} / \mathrm{N}$ & $\mathrm{Y} / \mathrm{N}$ & $\mathrm{Y} / \mathrm{N}$ & $\mathrm{Y} / \mathrm{N}$ & $\mathrm{Y} / \mathrm{N} \_$hours & $\mathrm{Y} / \mathrm{N} \_$Meds & $\mathrm{Y} / \mathrm{N} \_$Meds & $\mathrm{Y} / \mathrm{N} \_$mins./_ \\
\hline Intercostal Nerve & $\mathrm{Y} / \mathrm{N}$ & $\mathrm{Y} / \mathrm{N}$ & $\mathrm{Y} / \mathrm{N}$ & $\mathrm{Y} / \mathrm{N}$ & $\mathrm{Y} / \mathrm{N} \_$hours & $\mathrm{Y} / \mathrm{N} \_$Meds & $\mathrm{Y} / \mathrm{N} \_$Meds & $\mathrm{Y} / \mathrm{N} \_$mins./_ \\
\hline $\begin{array}{l}\text { Spinal Cord } \\
\text { Stimulator Trial }\end{array}$ & $\mathrm{Y} / \mathrm{N}$ & $\mathrm{Y} / \mathrm{N}$ & $\mathrm{Y} / \mathrm{N}$ & $\mathrm{Y} / \mathrm{N}$ & $\mathrm{Y} / \mathrm{N} \_$_hours & $\mathrm{Y} / \mathrm{N} \_$Meds & $\mathrm{Y} / \mathrm{N} \_$Meds & $\mathrm{Y} / \mathrm{N} \_$___ mins./_ \\
\hline $\begin{array}{l}\text { Intrathecal } \\
\text { Catheter Trial }\end{array}$ & $\mathrm{Y} / \mathrm{N}$ & $\mathrm{Y} / \mathrm{N}$ & $\mathrm{Y} / \mathrm{N}$ & $\mathrm{Y} / \mathrm{N}$ & $\mathrm{Y} / \mathrm{N} \_$hours & $\mathrm{Y} / \mathrm{N} \_$Meds & $\mathrm{Y} / \mathrm{N} \_$Meds & mins.I_ \\
\hline \multicolumn{9}{|c|}{ Have any of your patients experienced a Vasovagal Response during any of the above procedures within the last 12 months? If YES, how many? Y/N \# } \\
\hline \multicolumn{9}{|c|}{ Have any of your patients experienced a Syncopal Episode during any of the above procedures within the last 12 months? If YES, how many? Y/N \#__ } \\
\hline \multicolumn{9}{|c|}{ Have any of your patients "Coded" during any procedure above within the last 12 months? If YES, how many? } \\
\hline \multicolumn{9}{|c|}{ Do you routinely perform "mock Codes" or train your staff in Emergency Procedures? } \\
\hline
\end{tabular}

\section{Author Affiliation:}

Shihab U. Ahmed, MD, MPH

Massachusetts General Hospital Pain Center

WACC 324

15 Parkman Street

Boston, MA 021114

E-mail: shihablincoln@aol.com

William Tonidandel, DMD, MD

Attending Anesthesiologist

St. Peter's Hospital

Pain Specialist

Pain Specialists of Helena LLC

1 Medical Park Drive

Helena, MT 59601
Yuchiao Chang, PhD

Medical Practices Evaluation Center

Massachusetts General Hospital

Harvard Medical School

25 Shattuck Street

Boston, MA 02115
Massachusetts General Hospital

55 Fruit Street

Boston, MA 02114

Nicole M. Martin, BS

Harvard Medical School

25 Shattuck Street

Boston, MA 02115 


\section{References}

1. Fitzgibbon $\mathrm{DR}$, Posner $\mathrm{KL}$, Domino $\mathrm{KB}$, Caplan RA, Lee LA, Cheney FW. American Society of Anesthesiologists. Chronic pain management: American Society of Anesthesiologists Closed Claims Project. Anesthesiology 2004; 100:98-105.

2. Windsor RE, Pinzon EG, Gore HC. Complications of common selective spinal injections: prevention and management. $\mathrm{Am}$ J Orthopedics 2000; 29:759-770.

3. Houten JK, Errico TJ. Paraplegia after lum- bosacral nerve root block: report of three cases. Spine J 2002; 2:70-75.

4. McMillan MR, Crumpton C. Cortical blind ness and neurologic injury complicating cervical transforaminal injection for cervical radiculopathy. Anesthesiology 2003; 99:509-511.

5. Rozin L, Rozin R, Koehler SA, Shakir A, Ladham S, Barmada M, Dominick J, Wecht CH. Death during transforaminal epidural steroid nerve root block $\left(\mathrm{C}_{7}\right)$ due to perforation of the left vertebral artery. Am J Forensic Med Pathol 2003; 24:351-355.
6. Basic Standards for Pre-anesthesia Care. ASA House of Delegates, Oct. 14, 1987.

7. Standards for Basic Anesthetic Monitoring. ASA House of Delegates, Oct. 21, 1986.

8. Standards for Post-anesthesia Care. ASA House of Delegates Oct. 12, 1988.

9. Bogduk N. International Spinal Injection Society guidelines for the performance of spinal injection procedures. Part 1: Zygapophyseal joint blocks. Clin J Pain 1997; 13:285-302. 